\title{
Sex determination of non-model organisms in the absence of field records using Diversity Arrays Technology (DArT) data
}

\author{
Isabelle Onley ${ }^{1}$, Jeremy Austin ${ }^{1}$, and Kieren Mitchell ${ }^{2}$ \\ ${ }^{1}$ The University of Adelaide Faculty of Sciences \\ ${ }^{2}$ University of Adelaide Faculty of Sciences
}

October 1, 2020

\begin{abstract}
Conservation genomics research often relies on accurate sex information to make inferences about species demography, dispersal, and population structure. However, field determined sex data are not always available and can be subject to human error, while laboratory sex determination is costly and often challenging for non-model species. Conservation genomics programs increasingly use reduced-representation genome sequencing to assess neutral and functional genetic diversity, population structure, gene flow and pedigrees in threatened species. Here we demonstrate that sex can be determined from reduced-representation sequencing data produced by the increasingly popular Diversity Arrays Technology sequencing workflow (DArT-seq) using a program originally designed for application to shotgun data. This program - sexassign - compares the "dosage" of sequencing reads mapping to autosomes versus the $\mathrm{X}$ chromosome. In the present study, sexassign accurately determined the sex of 60 fieldcollected Greater Stick-Nest Rat (Leporillus conditor) samples, despite the absence of an annotated reference genome for the species. This "read-dosage" approach is not only more accurate and affordable than traditional sex determination methods, but can be applied to any diploid organism with a heterogametic sex determination system - including non-model and understudied species of conservation importance - by using FASTQs generated by DArT.
\end{abstract}

\section{Keywords}

Conservation genomics, sex determination, bioinformatics, DArT-seq

\section{Introduction}

Accurate sex determination is an integral aspect of conservation genomics research, particularly when studying parameters such as relatedness, dispersal, and philopatry. Sexing of individuals used in conservation genomics studies typically takes place in the field at the time of collection. However, sex assignments recorded in the field are not always reliable and there is a wide margin for human error, particularly for species that do not demonstrate sexual dimorphism or when researchers are working in difficult conditions. Further, field records can easily be lost or incorrectly transcribed during trapping and monitoring. Genetic sex determination is a favourable alternative or complement to field identification, as it is an objective, highly standardized, and accurate approach that eliminates the possibility of upstream sex misidentification confounding genomic studies (Hrovatin \& Kunej, 2017).

While PCR-based sex identification methods have been used for several decades to identify and amplify sex chromosomes in individual samples (Akane et al., 1992; Clapcote \& Roder, 2005; McFarlane et al., 2013), such processes can be time consuming and expensive. In addition, they require taxon-specific primers that are not always available or applicable to the target species. With the advent of high-throughput sequencing (HTS) technology it is now possible to produce high-resolution genomic data that may allow researchers to determine the sex of sequenced individuals bioinformatically. For example, single nucleotide polymorphisms (SNPs) in the genome can often be linked to the sex chromosomes in model organisms, allowing sex to be 
determined on chromosomal presence-absence basis (Fowler \& Buonaccorsi, 2016; Lambert et al., 2016). For non-model organisms where a well-assembled and well-annotated reference genome is unavailable, the overall "dosage" of sequencing reads mapping to the sex chromosomes can be assessed to determine whether the individual is heterogametic or homogametic (Bover et al., 2018; Gamble, 2016; Gower et al., 2019; Pečnerová et al., 2017).

Read-dosage-based approaches to sex determination have only been applied using shotgun sequencing data, where molecules are randomly sampled and sequenced (Flamingh et al., 2020; Motahari et al., 2013; Skoglund et al., 2013). However, many conservation programs employ reduced-representation sequencing approaches (e.g. RADseq), where sequenced molecules belong to a subset of genomic loci. One commercial provider of reduced-representation sequencing that is growing in popularity in the conservation genomics field is Diversity Arrays Technology (DArT) (Cummins et al., 2019; Ewart et al., 2019; Pazmiño et al., 2018; Sansaloni et al., 2011; Schultz et al., 2018; van Deventer et al., 2020). The DArT workflow uses restriction enzymes to reduce genomic complexity, allowing identification of informative markers that are subsequently sequenced for all submitted samples (Kilian et al., 2012). However, despite the growing popularity of DArT for conservation genomics projects, no simple and widely applicable sex-determination framework has emerged that can be applied to DArT data. In the present study we apply a read-dosage sex-determination approach to DArT data from an Australian rodent, the Greater Stick-Nest Rat (Leporillus conditor ), and demonstrate that despite being originally designed for application to shotgun data - this method remains robust when applied to FASTQ files generated as part of the DArT workflow.

\section{Materials and Methods}

DNA submitted to DArT was extracted from $60 \mathrm{~L}$. conditor tissue samples collected by staff during routine trapping events at Arid Recovery Reserve, South Australia, between 1999 and 2003. DNA extraction was completed following the methods described by Barclay et al. (2006) and samples were subsequently stored at $-20^{\circ} \mathrm{C}$ prior to sequencing by DArT. Following library preparation and sequencing by DArT using their proprietary workflow, we obtained the raw Illumina data in FASTQ format. We used the Paleomix v1.2.14 pipeline to process these data: AdapterRemoval2 v2.3.1 was used to trim residual adapter sequences (using default parameters) and filter reads shorter than $30 \mathrm{bp}$, after which all remaining reads were mapped against the repeat-masked house mouse genome assembly (GRCm38) using BWA v0.7.17 mem algorithm. We then used the idxstats command in SAMtools v1.10 to extract the number of reads mapping to each scaffold of the reference assembly.

We visualised read-dosage for each sample by using RStudio v1.3.1073 to plot the number of reads mapping to the autosomes and $\mathrm{X}$ chromosome (as a proportion of the total reads per sample) versus scaffold length. To determine the sex of the Greater Stick-Nest Rat samples we used Gower et al.'s (2019) python script sexassign(https://github.com/grahamgower/sexassign). This python script uses a likelihood ratio test to assign samples to either male or female on the basis of the observed ratio of reads mapping to the $\mathrm{X}$ chromosome versus the autosomes. However, because an underlying assumption of the calculations made by sexassign is that the $\mathrm{X}$ chromosome in homogametic females should receive the same read-dosage as an autosome of the same length (i.e. read dosage of $\sim 1 \mathrm{X}$ versus $\sim 0.5 \mathrm{X}$ in heterogametic males), we first multiplied the number of reads mapping to the $\mathrm{X}$ chromosome for all samples (regardless of putative sex) by a factor of 2.12 (the expected read-dosage for the $\mathrm{X}$ chromosome, 0.065 , divided by the observed mean read-dosage for the $\mathrm{X}$ chromosome in putatively female samples, 0.0308 , see Results).

\section{Results}

The proportion of reads mapping to each of the autosomes was highly consistent between samples (Fig. 1). Further, autosomal read-dosage appeared to be positively correlated with scaffold length, as expected if restriction motifs are randomly distributed. We tested this correlation by performing a linear regression in RStudio (proportion of reads ${ }^{\sim}$ scaffold length), which resulted in a slope coefficient of $3.833 \mathrm{e}^{-10}$ (adjusted $\left.\mathrm{R}^{2}=0.7, \mathrm{p}<2 \mathrm{e}^{-16}\right)$. Unlike the autosomes, values for the proportion of reads mapping to the $\mathrm{X}$ chromosome formed two clusters, putatively representing females (with higher read-dosage values) and males 
(with lower read-dosage values). However, the mean proportion of reads mapping to the $\mathrm{X}$ chromosome (length $=171,031,299 \mathrm{bp}$ ) for the putatively female samples $(0.0308)$ was substantially lower than the expectation (0.0656) based on the relationship between the proportion of reads mapped and scaffold length inferred from the autosomes. This difference may represent an inherent bias against sex-linked loci in the DArT pre-sequencing workflow or a depletion in the restriction motif on the $\mathrm{X}$ chromosome relative to the autosomes.

The read-dosage sex-assignment program (sexassign ) allowed us to successfully assign all individuals in the dataset as either male (heterogametic, $\mathrm{XY}$; $\mathrm{X}$ read-dosage $=\sim 0.5 \mathrm{X}$ ) or female (homogametic, $\mathrm{XX} ; \mathrm{X}$ read-dosage $=\sim 1 \mathrm{X}$, Fig. 2, Table 1 ). Of the 60 individuals sequenced, 33 were determined to be female and 27 to be male, consistent with the typical sex ratio in rodent populations under normal conditions (Labov et al., 1986; Rosenfeld et al., 2003). Genetic sex determination had a $\sim 94 \%$ concurrence rate with field determined sex, a typical human error margin considering the lack of obvious sexual dimorphism within the species and the difficulty of accurately sexing rodents in the field, particularly during non-reproductive periods (Hoffmann et al., 2010; Jacques et al., 2015).

\section{Discussion}

Our results demonstrate that the FASTQ-formatted data routinely generated by Diversity Arrays Technology (DArT) as an intermediate step in their workflow can reliably be used to determine the sex of samples from non-model organisms, confirming or replacing field-based sex identification and eliminating the need for additional costly laboratory sexing analyses. Importantly, a reference genome from the species of interest does not appear to be necessary, as we obtained robust results by mapping our data to the reference assembly for the house mouse (Mus musculus), which shared a common ancestor withL. conditor 10 million years ago (Steppan \& Schenk, 2017). While the house mouse genome is assembled to the chromosome-level, making identification of reads mapping to the $\mathrm{X}$ chromosome straightforward, this approach should also work with scaffold-level reference assemblies. Indeed, Gower et al. (2019) identified X-linked scaffolds in the polar bear genome (UrsMar1.0) by first mapping all scaffolds against the chromosome-level dog reference assembly (CanFam3.1), then appliedsexassign to shotgun sequencing data from a third species - brown bears (Ursus arctos ) - that they mapped to the putative polar bear X-linked scaffolds. Given that scaffold-level assemblies are increasingly available for a wide range of taxa, our results suggest that most DArT end-users working on mammals (or indeed any diploid organism with a heterogametic sex-determination system) should be able use their FASTQ data to determine the sex of their samples.

\section{Acknowledgements}

The authors wish to acknowledge Dr Katherine Moseby, Shaun Barclay, and the staff of Arid Recovery Reserve for supplying the field data and samples used in this study. This research was supported by the University of Adelaide and funded by the following organisations and awards; Australian Government Research Training Program Scholarship, Nature Foundation South Australia Grand Start Grant (Grant No. 2019-07), Biological Society South Australia/Nature Conservation Society of South Australia Conservation Biology Grant, Field Naturalists Society of South Australia Lirabenda Endowment Fund Research Grant.

\section{Conflict of Interest}

The authors declare no conflict of interest.

\section{References}

Akane, A., Seki, S., Shiono, H., Nakamura, H., Hasegawa, M., Kagawa, M., Matsubara, K., Nakahori, Y., Nagafuchi, S., \& Nakagome, Y. (1992). Sex determination of forensic samples by dual PCR amplification of an X-Y homologous gene. Forensic Science International , 52 (2), 143-148. https://doi.org/10.1016/03790738(92)90102-3

Bover, P., Llamas, B., Thomson, V. A., Pons, J., Cooper, A., \& Mitchell, K. J. (2018). Molecular resolution to a morphological controversy: The case of North American fossil muskoxen Bootherium and Symbos. 
Molecular Phylogenetics and Evolution, 129 , 70-76. https://doi.org/10.1016/j.ympev.2018.08.008

Clapcote, S. J., \& Roder, J. C. (2005). Simplex PCR assay for sex determination in mice. BioTechniques , 38 (5), 702-706. https://doi.org/10.2144/05385BM05

Cummins, D., Kennington, W. J., Rudin-Bitterli, T., \& Mitchell, N. J. (2019). A genome-wide search for local adaptation in a terrestrial-breeding frog reveals vulnerability to climate change. Global Change Biology , 25 (9), 3151-3162. https://doi.org/10.1111/gcb.14703

Ewart, K. M., Johnson, R. N., Ogden, R., Joseph, L., Frankham, G. J., \& Lo, N. (2019). Museum specimens provide reliable SNP data for population genomic analysis of a widely distributed but threatened cockatoo species. Molecular Ecology Resources , 19 (6), 1578-1592. https://doi.org/10.1111/1755-0998.13082

Flamingh, A. de, Coutu, A., Roca, A. L., \& Malhi, R. S. (2020). Accurate Sex Identification of Ancient Elephant and Other Animal Remains Using Low-Coverage DNA Shotgun Sequencing Data. G3: Genes, Genomes, Genetics , 10 (4), 1427-1432. https://doi.org/10.1534/g3.119.400833

Fowler, B. L. S., \& Buonaccorsi, V. P. (2016). Genomic characterization of sex-identification markers in Sebastes carnatus and Sebastes chrysomelas rockfishes. Molecular Ecology , 25 (10), 2165-2175. https://doi.org/10.1111/mec.13594

Gamble, T. (2016). Using RAD-seq to recognize sex-specific markers and sex chromosome systems. Molecular Ecology , 25 (10), 2114-2116. https://doi.org/10.1111/mec.13648

Gower, G. (2019). Inferring the Charactersitics of Ancient Populations using Bioinformatic Analysis of Genome-wide DNA Sequencing Data [Doctoral Dissertation]. University of Adelaide.

Gower, G., Fenderson, L. E., Salis, A. T., Helgen, K. M., van Loenen, A. L., Heiniger, H., Hofman-Kamińska, E., Kowalczyk, R., Mitchell, K. J., Llamas, B., \& Cooper, A. (2019). Widespread male sex bias in mammal fossil and museum collections. Proceedings of the National Academy of Sciences , 116 (38), 19019-19024. https://doi.org/10.1073/pnas.1903275116

Hoffmann, A., Decher, J., Rovero, F., Schaer, J., Voigt, C., \& Wibbelt, G. (2010). Field Methods and Techniques for Monitoring Mammals.Manual on Field Recording Techniques and Protocols for All Taxa Biodiversity Inventories , 8 , 482-529.

Hrovatin, K., \& Kunej, T. (2017). Genetic sex determination assays in 53 mammalian species: Literature analysis and guidelines for reporting standardization. Ecology and Evolution , 8 (2), 1009-1018. https://doi.org/10.1002/ece3.3707

Jacques, M.-E., McBee, K., \& Elmore, D. (2015). Determining Sex and Reproductive Status of Rodents . 4.

Kilian, A., Wenzl, P., Huttner, E., Carling, J., Xia, L., Blois, H., Caig, V., Heller-Uszynska, K., Jaccoud, D., Hopper, C., Aschenbrenner-Kilian, M., Evers, M., Peng, K., Cayla, C., Hok, P., \& Uszynski, G. (2012). Diversity Arrays Technology: A Generic Genome Profiling Technology on Open Platforms. In F. Pompanon \& A. Bonin (Eds.), Data Production and Analysis in Population Genomics: Methods and Protocols (pp. 67-89). Humana Press. https://doi.org/10.1007/978-1-61779-870-2_5

Labov, J. B., William Huck, U., Vaswani, P., \& Lisk, R. D. (1986). Sex ratio manipulation and decreased growth of male offspring of undernourished golden hamsters (Mesocricetus auratus). Behavioral Ecology and Sociobiology , 18 (4), 241-249. https://doi.org/10.1007/BF00300000

Lambert, M. R., Skelly, D. K., \& Ezaz, T. (2016). Sex-linked markers in the North American green frog (Rana clamitans) developed using DArTseq provide early insight into sex chromosome evolution. $B M C$ Genomics , 17 (1), 844. https://doi.org/10.1186/s12864-016-3209-x

McFarlane, L., Truong, V., Palmer, J. S., \& Wilhelm, D. (2013). Novel PCR Assay for Determining the Genetic Sex of Mice. Sexual Development , 7 (4), 207-211. https://doi.org/10.1159/000348677 
Motahari, A. S., Bresler, G., \& Tse, D. N. C. (2013). Information Theory of DNA Shotgun Sequencing. IEEE Transactions on Information Theory , 59 (10), 6273-6289. https://doi.org/10.1109/TIT.2013.2270273

Pazmiño, D. A., Maes, G. E., Green, M. E., Simpfendorfer, C. A., Hoyos-Padilla, E. M., Duffy, C. J. A., Meyer, C. G., Kerwath, S. E., Salinas-de-León, P., \& van Herwerden, L. (2018). Strong trans-Pacific break and local conservation units in the Galapagos shark (Carcharhinus galapagensis ) revealed by genome-wide cytonuclear markers.Heredity , 120 (5), 407-421. https://doi.org/10.1038/s41437-017-0025-2

Pečnerová, P., Díez-del-Molino, D., Dussex, N., Feuerborn, T., von Seth, J., van der Plicht, J., Nikolskiy, P., Tikhonov, A., Vartanyan, S., \& Dalén, L. (2017). Genome-Based Sexing Provides Clues about Behavior and Social Structure in the Woolly Mammoth. Current Biology ,27 (22), 3505-3510.e3. https://doi.org/10.1016/j.cub.2017.09.064

Rosenfeld, C. S., Grimm, K. M., Livingston, K. A., Brokman, A. M., Lamberson, W. E., \& Roberts, R. M. (2003). Striking variation in the sex ratio of pups born to mice according to whether maternal diet is high in fat or carbohydrate. Proceedings of the National Academy of Sciences , 100 (8), 4628-4632. https://doi.org/10.1073/pnas.0330808100

Sansaloni, C., Petroli, C., Jaccoud, D., Carling, J., Detering, F., Grattapaglia, D., \& Kilian, A. (2011). Diversity Arrays Technology (DArT) and next-generation sequencing combined: Genome-wide, high throughput, highly informative genotyping for molecular breeding of Eucalyptus. BMC Proceedings , 5 (S7), P54, 17536561-5-S7-P54. https://doi.org/10.1186/1753-6561-5-S7-P54

Schultz, A. J., Cristescu, R. H., Littleford-Colquhoun, B. L., Jaccoud, D., \& Frere, C. H. (2018). Fresh is best: Accurate SNP genotyping from koala scats. Ecology and Evolution , 8 (6), 3139-3151. https://doi.org/10.1002/ece3.3765

Skoglund, P., Stora, J., Gotherstrom, A., \& Jakobsson, M. (2013). Accurate sex identification of ancient human remains using DNA shotgun sequencing. Journal of Archaeological Science , 40 (12), 4477-4482. https://doi.org/10.1016/j.jas.2013.07.004

Steppan, S. J., \& Schenk, J. J. (2017). Muroid rodent phylogenetics: 900-species tree reveals increasing diversification rates. PLOS ONE , 12 (8), e0183070. https://doi.org/10.1371/journal.pone.0183070

van Deventer, R., Rhode, C., Marx, M., \& Roodt-Wilding, R. (2020). The development of genome-wide single nucleotide polymorphisms in blue wildebeest using the DArTseq platform. Genomics , 112 (5), 3455-3464. https://doi.org/10.1016/j.ygeno.2020.04.032

Data Accessibility Statement (to be archived upon acceptance)

The reads generated for this study have been deposited at the Sequence Read Archive (NCBI) with project number TBA

\section{Animal Ethics Statement}

Live animal trapping and sampling at Arid Recovery was conducted under South Australian Wildlife Ethics Committee permit number 58-2015.

\section{Author Contributions}

IRO and JJA coordinated submission of samples to DArT. IRO and KJM analysed the data. IRO drafted the abstract, introduction, results, and discussion. KJM drafted the materials and methods and figures. All authors contributed to the interpretation of results and provided feedback on the final manuscript.

Table 1. Results from sexassign, including sex assignment. The length of the $\mathrm{X}$ chromosome was 171,031,299 bp and the total length of the autosomes was 2,462,745,373 bp (Gower, 2019).

\begin{tabular}{lllll}
\hline ID $^{+}$ & $\mathbf{M}_{\mathbf{X}}{ }^{++}$ & Sex & $\mathbf{N}_{\mathbf{X}}{ }^{\S}$ & $\mathbf{N}_{\mathbf{A}}{ }^{\mathbf{I}}$ \\
\hline ET002 & 0.474 & M & 26392 & 802947
\end{tabular}




\begin{tabular}{lllll}
\hline ID & $\mathbf{M}_{\mathbf{X}}{ }^{++}$ & Sex & $\mathbf{N}_{\mathbf{X}}{ }^{\S}$ & $\mathbf{N}_{\mathbf{A}} \mathbf{I}$ \\
\hline ET101 & 0.962 & $\mathrm{~F}$ & 23290 & 349580 \\
ET102 & 0.480 & $\mathrm{M}$ & 12663 & 380070 \\
ET103 & 0.953 & $\mathrm{~F}$ & 25446 & 385924 \\
ET106 & 0.515 & $\mathrm{M}$ & 12856 & 359385 \\
ET119 & 0.930 & $\mathrm{~F}$ & 60581 & 941980 \\
ET133 & 0.963 & $\mathrm{~F}$ & 26462 & 396880 \\
ET146 & 0.517 & $\mathrm{M}$ & 8416 & 234263 \\
ET147 & 0.979 & $\mathrm{~F}$ & 26407 & 388782 \\
ET147B & 0.976 & $\mathrm{~F}$ & 12858 & 190022 \\
ET148 & 0.507 & $\mathrm{M}$ & 14526 & 412334 \\
ET149 & 1.020 & $\mathrm{~F}$ & 29618 & 417327 \\
ET151 & 0.975 & $\mathrm{~F}$ & 24507 & 362393 \\
ET152 & 1.002 & $\mathrm{~F}$ & 28024 & 402617 \\
ET153 & 0.970 & $\mathrm{~F}$ & 26335 & 391810 \\
ET154 & 0.946 & $\mathrm{~F}$ & 25894 & 395471 \\
ET155 & 0.4821 & $\mathrm{M}$ & 14054 & 420275 \\
ET157 & 1.026 & $\mathrm{~F}$ & 28484 & 399170 \\
ET158 & 0.950 & $\mathrm{~F}$ & 26525 & 403485 \\
ET162 & 0.503 & $\mathrm{M}$ & 13867 & 397200 \\
ET163 & 0.946 & $\mathrm{~F}$ & 24215 & 370137 \\
ET163B & 0.473 & $\mathrm{M}$ & 14299 & 436150 \\
ET17 & 0.942 & $\mathrm{~F}$ & 58158 & 892183 \\
ET173 & 0.956 & $\mathrm{~F}$ & 27789 & 419868 \\
ET176 & 0.938 & $\mathrm{~F}$ & 22451 & 346121 \\
ET177 & 0.952 & $\mathrm{~F}$ & 26275 & 398926 \\
ET18 & 0.905 & $\mathrm{~F}$ & 39676 & 635045 \\
ET183 & 0.495 & $\mathrm{M}$ & 13220 & 384279 \\
ET184 & 0.487 & $\mathrm{M}$ & 32640 & 966813 \\
ET185 & 1.010 & $\mathrm{~F}$ & 26952 & 384146 \\
ET186 & 0.473 & $\mathrm{M}$ & 28294 & 863292 \\
ET187 & 0.996 & $\mathrm{~F}$ & 25964 & 375434 \\
ET188 & 0.503 & $\mathrm{M}$ & 12563 & 359345 \\
ET189 & 0.972 & $\mathrm{~F}$ & 22913 & 339929 \\
ET192 & 0.500 & $\mathrm{M}$ & 14444 & 416297 \\
ET193 & 0.489 & $\mathrm{M}$ & 13108 & 386485 \\
ET195 & 0.960 & $\mathrm{~F}$ & 25194 & 378761 \\
ET196 & 0.977 & $\mathrm{~F}$ & 27030 & 398915 \\
ET198 & 0.512 & $\mathrm{M}$ & 28970 & 813496 \\
ET198B & 0.484 & $\mathrm{M}$ & 12733 & 378965 \\
ET203 & 0.475 & $\mathrm{M}$ & 11469 & 348138 \\
ET209 & 0.971 & $\mathrm{~F}$ & 25533 & 379373 \\
ET217 & 0.480 & $\mathrm{M}$ & 13460 & 404344 \\
ET231 & 0.493 & $\mathrm{M}$ & 12745 & 372720 \\
ET233 & 0.480 & $\mathrm{M}$ & 12353 & 370852 \\
ET255 & 0.952 & $\mathrm{~F}$ & 24282 & 368459 \\
ET259 & 0.478 & $\mathrm{M}$ & 11357 & 342534 \\
ET261 & 0.958 & $\mathrm{~F}$ & 26557 & 400394 \\
ET2 & 0.488 & $\mathrm{M}$ & 29029 & 857827 \\
& 0.939 & $\mathrm{~F}$ & 30250 & 465742 \\
ET9 & $\mathrm{F}$ & 23511 & 354200
\end{tabular}




\begin{tabular}{lllll}
\hline ID $^{+}$ & $\mathbf{M}_{\mathbf{X}}{ }^{++}$ & Sex & $\mathbf{N}_{\mathbf{X}}{ }^{\S}$ & $\mathbf{N}_{\mathbf{A}} \mathbf{I}$ \\
\hline ET3 & 0.991 & $\mathrm{~F}$ & 27316 & 397077 \\
ET32 & 0.509 & $\mathrm{M}$ & 13059 & 369309 \\
ET37 & 0.467 & $\mathrm{M}$ & 26816 & 828029 \\
ET5 & 0.491 & $\mathrm{M}$ & 27564 & 809456 \\
ET50 & 0.485 & $\mathrm{M}$ & 11802 & 350729 \\
ET50.2 & 0.981 & $\mathrm{~F}$ & 23708 & 348582 \\
ET5967 & 0.958 & $\mathrm{~F}$ & 26131 & 393719 \\
ET61 & 0.491 & $\mathrm{M}$ & 7566 & 222226 \\
ET62 & 0.987 & $\mathrm{~F}$ & 36015 & 526076 \\
\hline
\end{tabular}

$+\mathrm{ID}=$ ear tag number for $L$. conditor individual, $++\mathrm{M}_{\mathrm{X}}=$ read dosage on $\mathrm{X}$ chromosome, $\S_{\mathrm{X}}=$ count of reads mapped to the $\mathrm{X}$ chromosome (after multiplying by 2.12), $\mathbb{I} \mathrm{N}_{\mathrm{A}}=$ count of reads mapped to the autosome.

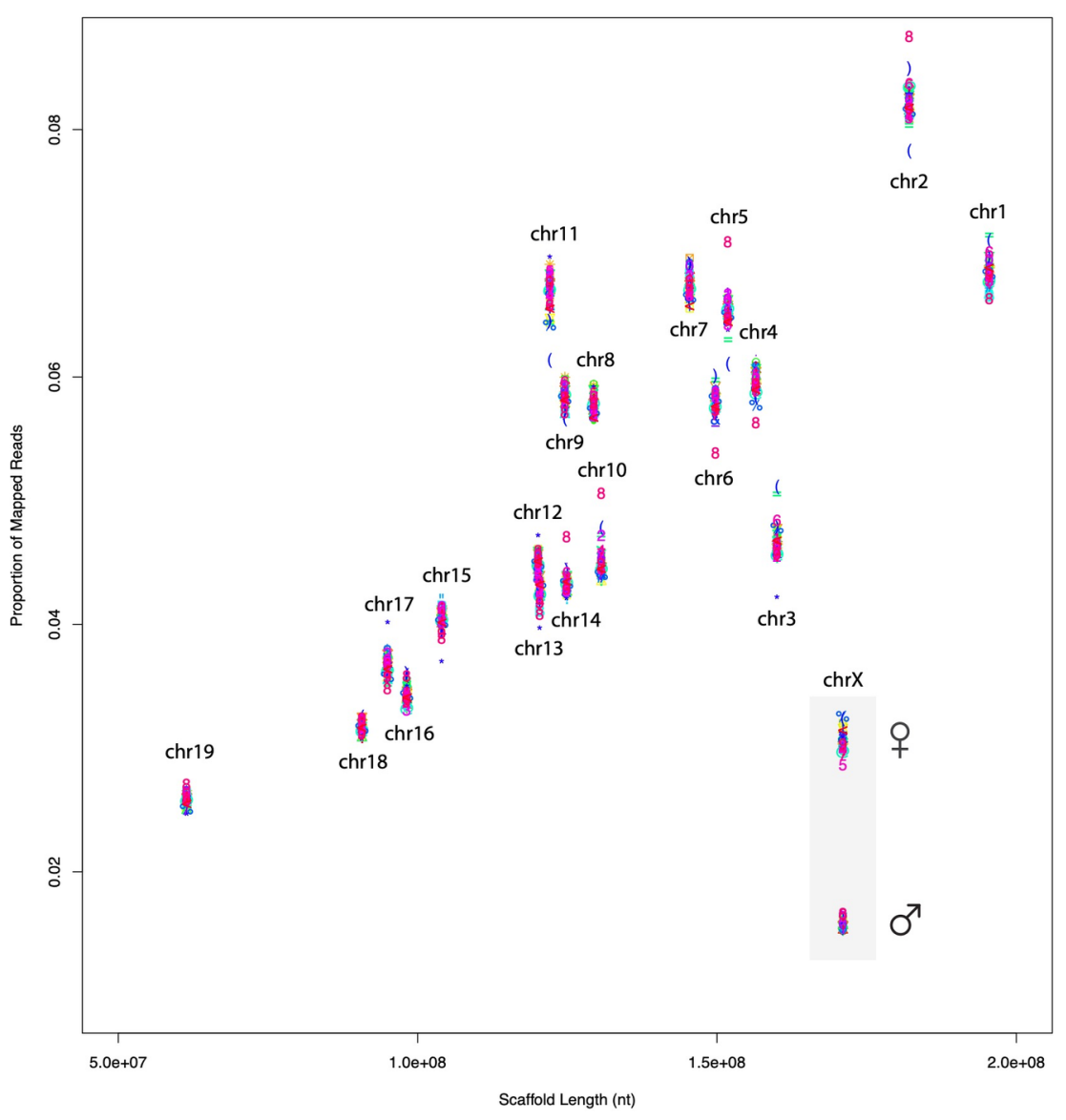

\section{Hosted file}

image2.emf available at https://authorea.com/users/363640/articles/484346-sex-determinationof-non-model-organisms-in-the-absence-of-field-records-using-diversity-arrays- 
technology-dart-data

Figure 2. Plot of $\mathrm{X}$ chromosome read dosages for all sequenced $L$. conditor individuals, with confidence intervals for male heterogametes (red) and female homogametes (blue). 